\title{
Theoretical Analysis of Tablet Hardness Prediction Using Chemoinformetric Near-Infrared Spectroscopy
}

\author{
Hideaki TAnabe, ${ }^{*}$ Kuniko OTsuKa, ${ }^{* *}$ and Makoto OTSUKA***† \\ * Kobe Pharmaceutical University, Motoyamakitamachi 4-17-1, Higashinada, Kobe 658-8558, Japan \\ **Department of Pharmacology, School of Medicine, Showa University, \\ Hatanodai 1-5-8, Shinagawa, Tokyo 142-8855, Japan \\ ***Research Institute of Pharmaceutical Sciences, Faculty of Pharmacy, Musashino University, \\ Shinmachi 1-1-20, Nishi-Tokyo, Tokyo 202-8585, Japan
}

\begin{abstract}
In order to clarify the theoretical basis of the variability in the measurement of tablet hardness by compression pressure, NIR spectroscopic methods were used to predict tablet hardness of the formulations. Tablets ( $200 \mathrm{mg}, 8 \mathrm{~mm}$ in diameter) consisting of berberine chloride, lactose, and potato starch were formed at various compression pressures $(59,78,98,127$, $195 \mathrm{MPa}$ ). The hardness and the distribution of micropores were measured. The reflectance NIR spectra of various compressed tablets were used as a calibration set to establish a calibration model to predict tablet hardness by principal component regression (PCR) analysis. The distribution of micropores was shifted to a smaller pore size with increasing compression pressure. The total pore volume of tablets decreased as the compression pressure increased. The hardness increased as the compression pressure increased. The hardness could be predicted using a calibration model consisting of 7 principal components (PCs) obtained by PCR. The relationship between the predicted and the actual hardness values exhibited a straight line, an $R^{2}$ of 0.925 . In order to understand the theoretical analysis (scientific background) of calibration models used to evaluate tablet hardness, the standard error of cross validation (SEV) values, the loading vectors of each PC and the regression vector were investigated. The result obtained with the calibration models for hardness suggested that the regression vector might involve physical and chemical factors. In contrast, the porosity could be predicted using a calibration model composed of 2 PCs. The relationship between the predicted and the actual total pore volume showed a straight line with $R^{2}=0.801$. The regression vector of the total pore volume might be due to physical factors.
\end{abstract}

(Received February 28, 2007; Accepted April 16, 2007; Published July 10, 2007)

\section{Introduction}

Since the introduction of guidelines for process analytical technology (PAT) by the Food and Drug Administration, online, real-time analyses as a tool for monitoring and controlling manufacturing processes have become increasingly accepted in the pharmaceutical industry. ${ }^{1-3}$ On-line analyses hold the promise of reducing or eliminating reworked batches, increasing manufacturing efficiency, decreasing the burden of finished product testing, and ensuring product quality throughout the manufacturing process. A substantial amount of time can be saved, and sampling difficulties minimized, by following reactions and processes in-situ composed for collecting samples and analyzing them in the laboratory with conventional approaches.

Near infrared (NIR) spectra can be measured directly on the surface of intact samples without any pretreatment. ${ }^{4}$ Consequently, NIR spectroscopy is rapidly becoming an important technique in process analytical methods of the pharmaceuticals industry. Chemoinformetrical NIR spectroscopic methods ${ }^{5}$ have been used to determine drug content, ${ }^{6}$ drug stability, ${ }^{7}$ the polymorphic content of

$\dagger$ To whom correspondence should be addressed.

E-mail: motsuka@musashino-u.ac.jp pharmaceuticals, ${ }^{8-12}$ as well as the particle size of powders. ${ }^{13-18}$

The measurement of tablet hardness and predictions are therefore necessary for quality control in the pharmaceutical industry, since sufficient mechanical strength of the preparations is important during both the manufacturing process and transport. There are several reports on measurements of tablet mechanical strength using the NIR method. ${ }^{19-23}$ However, in most of the research, predictions were not made on a theoretical basis concerning the tablet geometrical structure. Many factors contribute to the hardness of tablets, such as the tablet's porosity, the contact surface area of its particles, the cohesive force of the particle surface, the particle size distribution, the water content, and the characteristics of lubricants etc. These can be separated into physical factors, such as porosity, contact surface area of the particles, and particle size, as well as chemical factor, such cohesive force's due to the characteristics and concentration of a binder, and the composition of the tablet.

To clarify the theoretical background of chemoinformatic calibration models, the mixing effect of lubricants on the tablet hardness was investigated by NIR spectroscopy. ${ }^{23}$ Tablet hardness was predicted based on loading vectors and their scores, reflecting molecular interactions between lubricant and excipient surfaces from the spectra of the raw material powders, since the peaks due to hydrogen groups of excipients in the regression vector appeared as positive, whereas the peaks 


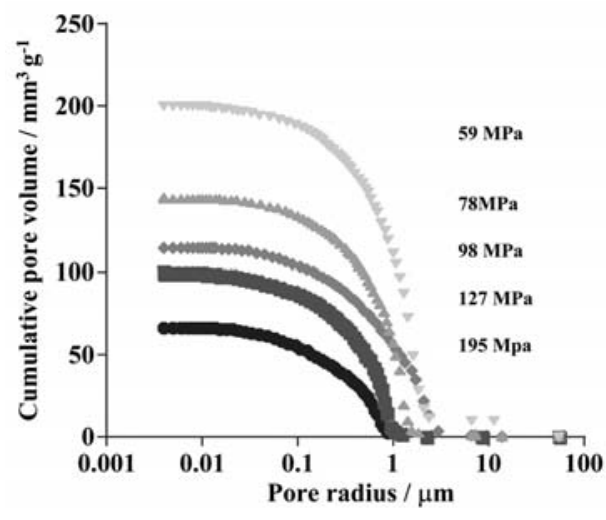

Fig. 1 Micropore distribution of compressed tablets.

related to the hydrocarbon of a lubricant were negative. The result suggested that chemoinformetrical analysis is able to clarify the chemical interaction in complex systems, such as pharmaceutical formulations. However it was not considered that the mechanical strength of a tablet was affected by both the physical and chemical properties.

In this study, in order to clarify the theoretical basis (geometrical structural factors and chemical interaction) of the variability in measurements of the tablet hardness compression pressure, the variable regulated to the tablet hardness was separated into physical and chemical factors by using chemoinformetrics, then NIR spectroscopic methods were applied to the compression properties of tablet formulations during the manufacturing process as a basic study of PAT.

\section{Materials and Methods}

\section{Materials}

A bulk powder of berberine chloride (Japanese Pharmacopoeia XIV grade) was obtained from ALPS Pharmaceutical Ind. Co. Ltd. (Gifu, Japan). Crystalline $\alpha$-lactose monohydrate (Pharmatose 200M, DeMelkindustrie Veghel Co. Veghel, Netherlands) and potato starch (Astra Japan, Ltd., Osaka, Japan) were used as diluents.

\section{Tableting compression process}

The manufacturing process for tablets was as follows: $2.5 \mathrm{~g}$ of berberine chloride bulk powder, $15.75 \mathrm{~g}$ of lactose, and $6.75 \mathrm{~g}$ of potato starch were mixed in a twin-shell mixer (Model V-1, capacity $2 \mathrm{~L}$, mixing speed $28 \mathrm{rpm}$, Tokujyu Ind. Co. Tokyo, Japan) for $20 \mathrm{~min}$. A compression/tension tester (Autograph, Model IS-5000, Shimadzu Co., Kyoto, Japan) was used at $25 \pm 1{ }^{\circ} \mathrm{C}$. An $8 \mathrm{~mm}$ diameter flat-surface punch and a die compressed mixture of $200 \mathrm{mg}$ were used at various compression pressures $(59,78,98,127,195 \mathrm{MPa})$ with a compression speed of $15 \mathrm{~mm} / \mathrm{min}$.

\section{Determination of hardness of tablet}

The hardness of each scanned tablet was determined using a tablet tester (Toyama Co. Osaka, Japan).

\section{Scanning electron microscopy (SEM)}

SEM images of samples were taken with a Model JSM5200LV (Jeol Co., Tokyo, Japan) microscope at a magnification of $\times 500$.
Table 1 Effect of the compression pressure on the hardness and total pore volume of tablets

\begin{tabular}{cccc}
\hline \multirow{2}{*}{$\begin{array}{c}\text { Compression } \\
\text { pressure/MPa }\end{array}$} & \multicolumn{2}{c}{ Hardness/kg } & \multirow{2}{*}{$\begin{array}{c}\text { Total pore } \\
\text { volume } / \mathrm{mm}^{3} \mathrm{~g}^{-1}\end{array}$} \\
\cline { 2 - 3 } & Ave. & S.D. & \\
\hline 59 & 1.6 & 0.12 & 200.1 \\
78 & 2.7 & 0.19 & 144.6 \\
98 & 3.6 & 0.11 & 114.2 \\
127 & 4.8 & 0.16 & 100.0 \\
195 & 5.7 & 0.25 & 65.7 \\
\hline
\end{tabular}

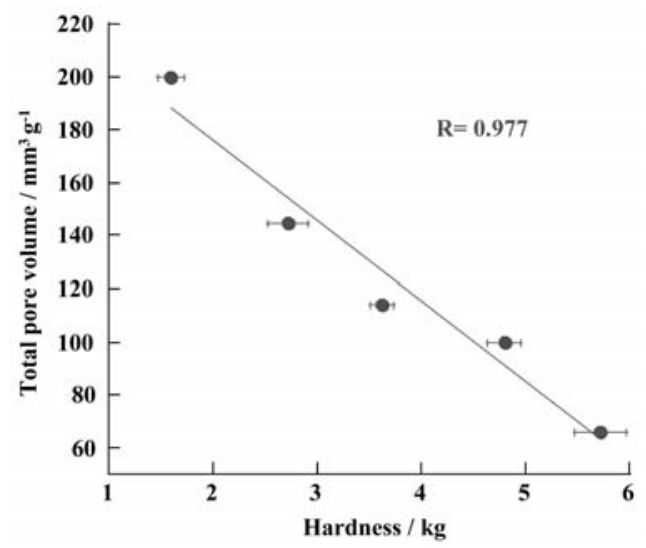

Fig. 2 Relationship between the total pore volume and the actual tablet hardness.

\section{Micropore distribution measurements}

The micropore distribution of tablets was measured by mercury porosimetry (type 2000 Carlo Erba Co. Ltd., Strumentazione, Italy). The contact angle and surface tension of mercury were 141.3 degrees and 480 dyne/cm, respectively. The pore radius ranged from $6 \times 10^{-3}$ to $300 \mu \mathrm{m}$.

\section{NIR spectroscopic measurements}

Reflectance NIR spectra were recorded with a NIR spectrometer (InfraProver II, BRAN + LUEBBE Co., Norderstedt, Germany). NIR spectra of 20 sample tablets (5 compression pressure levels, 4 tablets each) were recorded on the top and wire sides of the tablet by a reflectance NIR spectrometer. The 40 NIR spectra of 20 sample sets were used as a calibration set to establish a calibration model to predict tablet hardness by principal component regression (PCR) analysis. A chemometric analysis was performed using the PCR program associated with the Pirouette software (InfoMetrix Co., USA). The conditions were optimized to minimize the standard error of cross-validation (SEV). Each NIR spectrum including $n$ spectral data can be seen as a point in an $n$-dimensional space. In the multivariate data analysis, principal component analysis (PCA)/PCR of a spectral data matrix $\mathbf{X}$ is a basic tool. PCA/PCR decomposes $\mathbf{X}$ into a score matrix $\mathbf{T}$ times a loading matrix $\mathbf{P}$ plus a residual matrix $\mathbf{E}$ (Eq. $(1)),{ }^{24}$

$$
\mathbf{X}=t_{1} p^{\prime}{ }_{1}+t_{2} p^{\prime}{ }_{2}+\cdots+\mathbf{E}=\mathbf{T P}+\mathbf{E}
$$

This decomposition is particularly useful for converting $\mathbf{X}$ to a few information plots (score plots and loading plots) and for modeling the systematic structure in $\mathbf{X}$. 


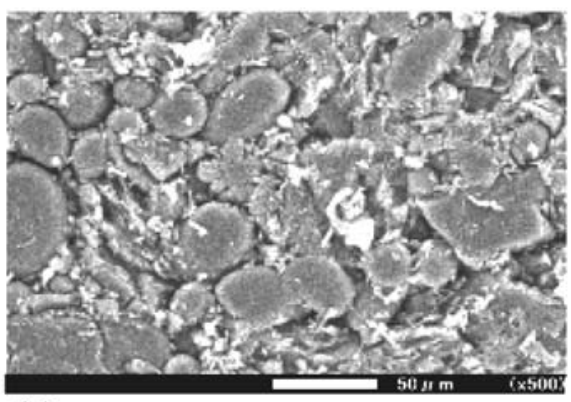

(a)

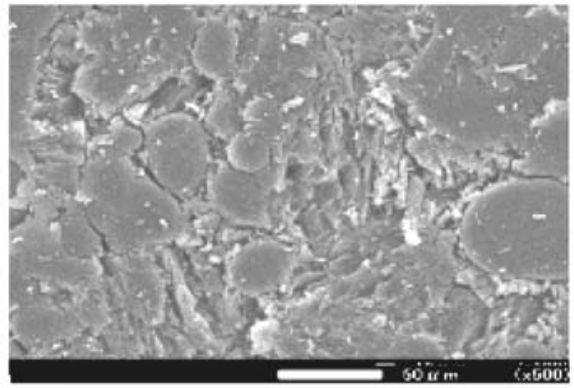

(c)

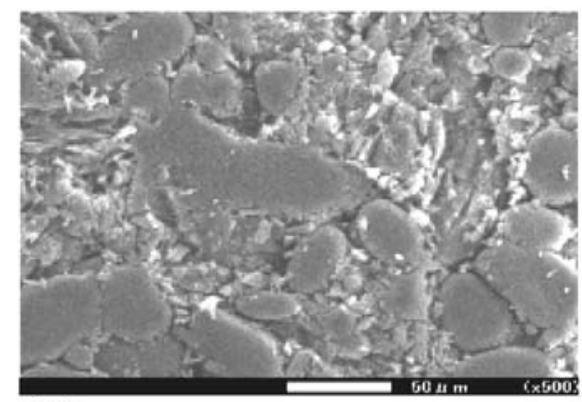

(b)

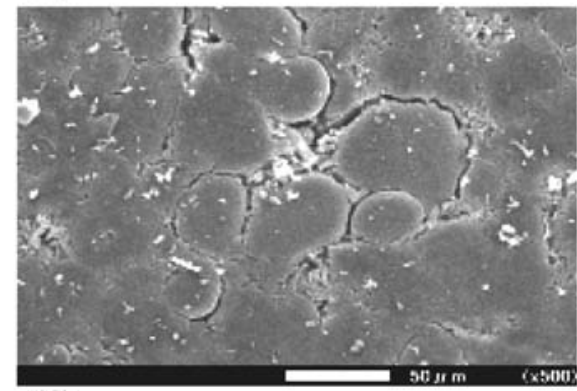

(d)

Fig. 3 SEM micrographs of the surface. Compression pressure: (a) 59 (b) 98, (c) 127, (d) $195 \mathrm{MPa}$.

\section{Results and Discussion}

Relationship between physical properties of tablet and compression pressure

Figure 1 shows the effect of compression pressure on the distribution of micropores in the tablets measured by mercury porosimetry. The distribution shifted to smaller pore size range as the compression pressure increased. The tablet hardness and the total pore volume are summarized in Table 1 . The tablet compressed at $59 \mathrm{MPa}$ had the highest total pore volume, while that compressed at $195 \mathrm{MPa}$ had the lowest. The total pore volume decreased as the compression pressure increased. The tablet hardness increased as the compression pressure increased, because the porosity decreased and the contact area between the particle surfaces increased.

Figure 2 shows the relationship between the hardness and the total pore volume. The plot gives a straight line with a correlation coefficient constant of 0.977 . The result suggested that the total pore volume governed the hardness of the tablets, meaning that the contact area between the particle surfaces increased as the compression pressure increased.

The geometrical structure of the tablet was evaluated by SEM. Figure 3 shows SEM micrographs of the surface. The tablet compressed at $59 \mathrm{MPa}$ had many pores and particles, whereas that compressed at $195 \mathrm{MPa}$ had a flat, smooth surface. The roughness of the surface decreased with increasing the compression pressure.

\section{NIR spectra of tablets compressed at various compression} pressures

Figure 4 shows the raw NIR spectra of the surfaces of tablets obtained at various compression pressures using the diffused reflectance methods. The absorbance of NIR increased in a regular fashion in response to an increase in the compression pressure. Chemoinformetrics can be used to decompose raw data profiles and help us to understand the significant contributions of specific data group. Quantitative relationships

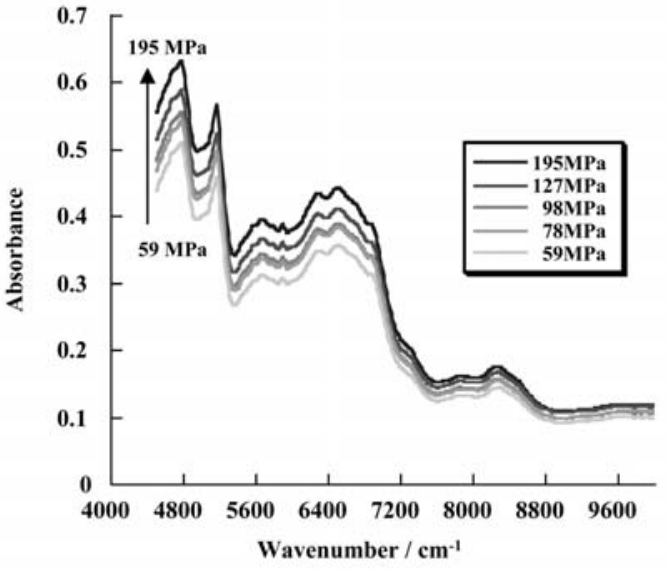

Fig. 4 Raw NIR spectra of the surface of tablets.

between the objective parameters, such as the pharmaceutical properties of tablets and the loadings of the principle components, were obtained from the NIR spectral results.

In the present study, in order to predict the tablet hardness, calibration models were established based on NIR spectra of the tablets and their actual hardness by PCR. Since raw absorbance scans show a baseline shift in the spectra corresponding to the compression pressure, the calibration model for the tablet hardness was based on the absorbance spectra without any pretreatment.

A calibration model consisting of 7 principal components (PCs) to predict tablet hardness was developed by PCR. The relationship between the predicted and actual hardness values are shown in Fig. 5, and the plot gives a straight line, with a slope of 0.922 , a $Y$-intercept of 0.282 , and a multiple correlation coefficient constant, $R^{2}$, of 0.925 . These predicted values were all within the $95 \%$ predictive intervals, indicating that NIR has a good predictive potential for tablet hardness. 


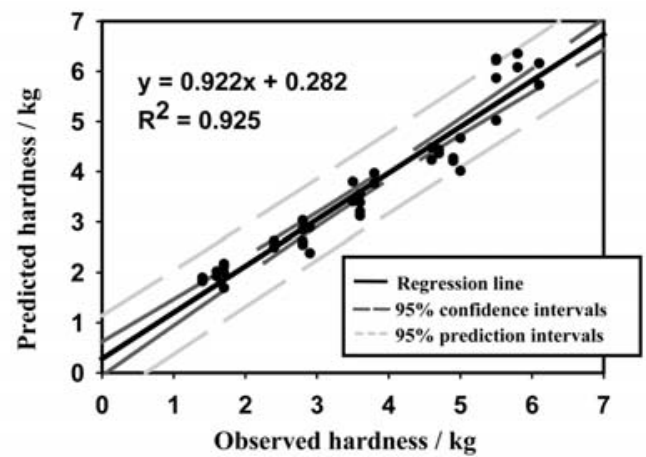

Fig. 5 Relationship between the actual and predicted tablet hardness $(n=40)$.

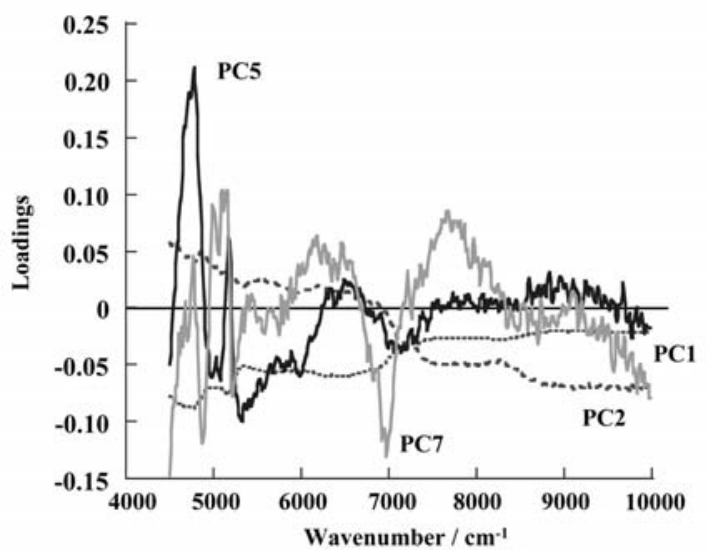

Fig. 6 Loading plots for predicting tablet hardness.

\section{Theoretical analysis of tablet hardness prediction}

Chemoinformetrics can be used to decompose raw data profiles and helps us to understand the significant contributions of specific data groups of process variables to the variability in raw data profiles. The quantitative relationships between the objective parameters, such as the pharmaceutical properties of tablets, and loadings of the PCs were thus obtained from the NIR spectral results as reported previously. ${ }^{23}$

On the other hand, the mechanical strength of the tablet was affected by the geometrical structure, such as the particle surface area and the characteristics of the surface, such as the bonding strength per unit area. Since the former factor was related to the tablet porosity and the latter to the hydrogen bonding network between interparticles, the variability of the tablet hardness during the manufacturing processes consisted of both physical and chemical factors. In the present study, in order to understand the theoretical analysis (scientific background) of the calibration models used to evaluate the tablet hardness with the diffused reflectance methods, the relationships between the SEV values and the number of used PCs, the loading vectors of each PC and the relationships between the scores of each PC and pharmaceutical properties of tablets were investigated.

In Table 2, the SEV value of the calibration model for hardness is 1.145 at PC1, 0.851 at PC2, 0.548 at PC5, and 0.403 at PC7. From this, it was recognized that the SEV values used to predict the hardness decreased greatly using PC1, PC2, PC5 and PC7.

Table 3 shows multiple correlation coefficients of the plots of the scores of PC against the pharmaceutical properties related to
Table 2 SEV and variance values in case of the tablet hardness and the total pore volume prediction

\begin{tabular}{ccccc}
\hline \multirow{2}{*}{$\begin{array}{c}\text { Number of } \\
\text { used principal } \\
\text { components }\end{array}$} & \multicolumn{2}{c}{$\begin{array}{c}\text { Tablet hardness/ } \\
\mathrm{kg}\end{array}$} & \multicolumn{2}{c}{$\begin{array}{c}\text { Total pore volume/ } \\
\mathrm{mm}^{3} \mathrm{~g}^{-1}\end{array}$} \\
\cline { 2 - 5 } & SEV & Variance, \% & SEV & Variance, \% \\
\hline PC1 & 1.145 & 97.725 & 30.536 & 97.053 \\
PC2 & 0.851 & 1.935 & 20.235 & 2.627 \\
PC3 & 0.888 & 0.138 & 20.853 & 0.143 \\
PC4 & 0.825 & 0.081 & 20.012 & 0.085 \\
PC5 & 0.548 & 0.062 & 19.740 & 0.036 \\
PC6 & 0.544 & 0.029 & 19.176 & 0.023 \\
PC7 & 0.403 & 0.010 & 17.460 & 0.012 \\
PC8 & 0.393 & 0.005 & 17.868 & 0.005 \\
PC9 & 0.398 & 0.003 & 14.756 & 0.003 \\
\hline
\end{tabular}

Table 3 Multiple correlation coefficient constants of plots of the score of PC against pharmaceutical properties

\begin{tabular}{cccc}
\hline $\begin{array}{c}\text { Principal } \\
\text { component }\end{array}$ & $\begin{array}{c}\text { Compression } \\
\text { pressure }\end{array}$ & Hardness & $\begin{array}{c}\text { Total pore } \\
\text { volume }\end{array}$ \\
\hline PC1 & 0.9532 & 0.8821 & 0.9476 \\
PC2 & 0.9296 & 0.7919 & 0.7949 \\
PC3 & 0.0021 & 0.0004 & 0.0127 \\
PC4 & 0.0641 & 0.0544 & 0.1095 \\
PC5 & 0.3445 & 0.4198 & 0.3474 \\
PC6 & 0.0102 & 0.0009 & 0.0051 \\
PC7 & 0.4455 & 0.6898 & 0.6498 \\
PC8 & 0.0150 & 0.0033 & 0.0150 \\
PC9 & 0.1154 & 0.0011 & 0.1154 \\
\hline
\end{tabular}

hardness. For all pharmaceutical properties, PC1 and PC2 and PC5 and PC7 had a better correlation than the other PCs. This result might indicate that the scores of PC1, PC2, PC5 and PC7 were closely related to pharmaceutical properties related to the hardness.

Figure 6 shows the loading vector of PC1, PC2, PC5 and PC7 which is closely related to the pharmaceutical properties related to the hardness. The loading vector of PC1 reached a plateau at around -0.05 . Therefore, the loading vector of $\mathrm{PC} 1$ might be considered as a baseline shift. On the other hand, the loading vector of PC2 showed positive peaks at lower wavenumbers, and negative peaks at higher wavenumbers, indicating that the intensity was inversely proportional to the wavenumber. The pattern of PC2 could be considered to be the slope of the baseline. It is well recognized that a spectral background shift is related to the particle size of samples in the diffuse reflectance method. ${ }^{13-18}$ Since Otsuka et al..$^{17,18}$ reported that a background shift was related to a large particle size, more than $50 \mu \mathrm{m}$ in diameter, the loading vector of PC1 might be related to the particle size or the radius of pores in the tablets. On the other hand, Norris and Williams ${ }^{16}$ reported that the slope of the spectral background was proportional to increasing particle size of a fine powder. The loading vector of PC2 indicated the relationship between the slope and the particle or pore size. The scores of PC1 and PC2 had a linear relationship with the total pore volume, as shown in Table 3. Therefore, the result of the diffused reflectance suggested that the calibration model consisted of loading vectors of PC1 and PC2 attributable to physical factors related to the particle size and/or pore volume and their pore radius in the sample tablet. Otsuka et al..$^{25}$ proposed a relationship between a physical model reflecting the 


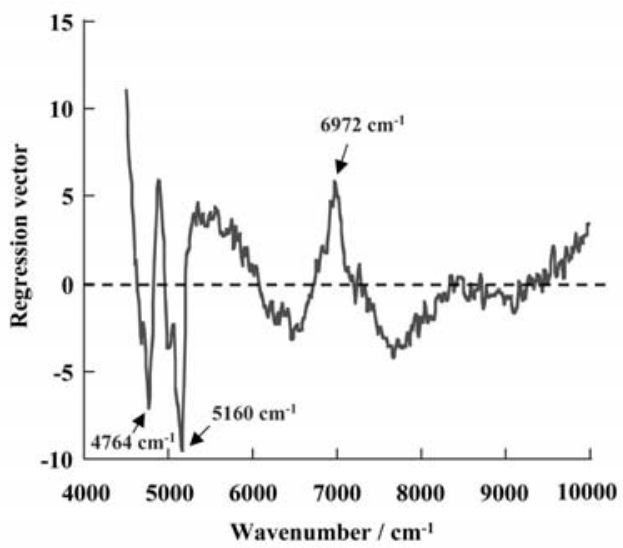

Fig. 7 Regression vector for predicting tablet hardness.

geometrical structure of a tablet and the light path-way. The background of the diffused reflectance spectra increased with decreasing micropore volume of the tablet due to compression pressure.

In contrast, for the main peaks, the loading vector of PC5 had positive peaks at 4776 and $5172 \mathrm{~cm}^{-1}$ that might be attributed to a combination of $\mathrm{OH}$ stretching and $\mathrm{OH}$ deformation. PC7 had a negative peak at $6972 \mathrm{~cm}^{-1}$ that might be attributed to the first overtone of $\mathrm{OH}$ stretching. The tablet hardness reflected the chemical factor related to interparticle bonding based on intermolecular hydrogen bonding between interparticles surfaces of the components of the tablet. Therefore, PC5 and PC7 might be the principal components reflecting the influence of hydrogen bonds between the particles. On the other hand, Table 2 shows that the value of SEV almost did not have any change when using PC3, PC4 and PC6. In addition, the multiple coefficient correlation of the plots of the scores of PC3, PC4 and PC6 against the pharmaceutical properties related to hardness shows a remarkably low value in Table 3. Since these results might suggest that PC3, PC4 and PC6 were not related to any pharmaceutical properties related to hardness, it thought that these PC were the component that reflected the factor that did not give any influence to the precision of the calibration model.

In general, the objective function $\hat{y}_{\mathrm{u}}$ could be calculated from the original spectral data and regression vector, $\beta .^{26}$ Therefore, the regression vector indicates important factors concerning the dissolution behavior in the NIR spectra of tablets.

$$
\hat{y}_{\mathrm{u}}=\beta \cdot X_{\mathrm{u}}
$$

where $\beta$ is the regression vector, and $X_{\mathrm{u}}$ and $y_{\mathrm{u}}$ are the test sample data vector and its predicted property, respectively.

Figure 7 shows the regression vector of the calibration models for hardness. The regression vector might be considered to be combinations of physical information of some baseline shifts related to geometrical changes of the tablets in the lowwavenumber region and chemical information related to the tablet formulation as mentioned above. However, the chemical information due to hydroxyl functional groups more clearly appeared in the regression vector.

From the above-mentioned discussion, the calibration model consists of first and second components to predict the total pore volume of tablets by PCR. In Table 2, the SEV values of the calibration model for the total pore volume are 20.235 at PC2. The SEV value did not decrease very much even if we increased the numbers using a principal component of more than PC2.

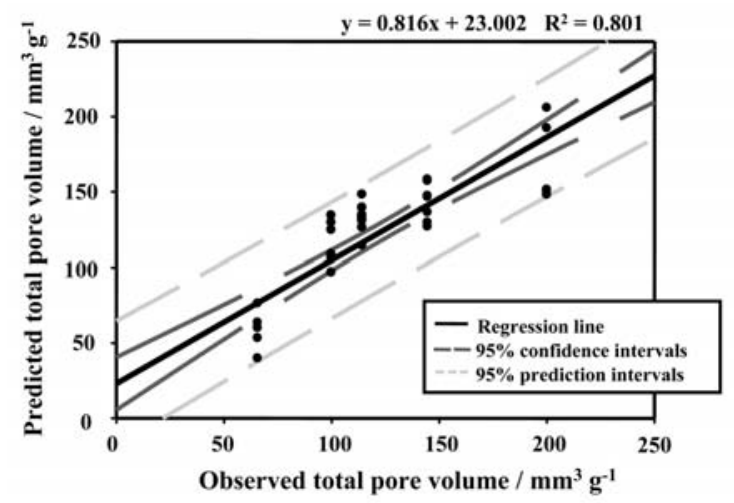

Fig. 8 Relationship between the actual and predicted total pore volume of tablets $(n=40)$.

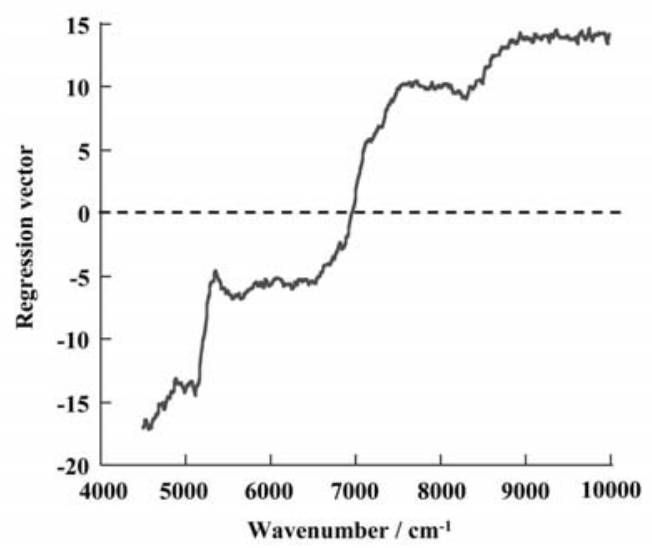

Fig. 9 Regression vector for predicting the total pore volume of tablets.

Figure 8 shows plots for the relationship between the predicted and actual total pore volume. The plot shows a straight line with a slope of 0.816 , a $Y$-intercept of 23.002 , and a multiple correlation coefficient constant, $R^{2}$, of 0.801 . These predicted values were all within the $95 \%$ predictive intervals, indicating that NIR has a good predictive potential for total pore volume.

Figure 9 shows the regression vector of the calibration models for the total pore volume. The regression vector of the total pore volume was the spectrum pattern that resembled the loading vector of PC2 in hardness prediction. The regression vector showed that the slope of the spectral background consisted of the first and second components in the NIR spectra reported by Norris and Williams, ${ }^{16}$ indicating that increasing of the slope might be related to the particle and/or pore size of the tablets, since the regression vector might be involved in the loading vectors of PC1 and PC2. The regression vector for the total pore volume might be reflected only by the physical information on the tablet. Since the total pore volume was related to the geometrical structure of the tablet, such as the particle surface area and the characteristics of the surface, it was thought that we would be able to obtain a good calibration line in this condition.

\section{Conclusion}

Calibration models used to evaluate tablet hardness were 
obtained based on diffused reflectance NIR spectra by using PCR. It was suggested that the tablet hardness could quickly and nondestructively be predicted based on the NIR spectra. By analyzing a calibration model theoretically based on geometrical structural factors of the tablet and the chemical interaction between fine particles, the result obtained with the calibration models for hardness suggested that the regression vector might involve both physical and chemical factors. In contrast, the porosity could be predicted using a calibration model composed of 2 PCs. The regression vector of the total pore volume might be due to physical factors.

\section{Acknowledgements}

This work was supported in part by a Grant-(Scientific Research, C, No. 17500322) in-Aid for Scientific Research and HAITEKU (2004-2008) from the Ministry of Education, Culture, Sports, Sciences and Technology, Japan.

\section{References}

1. W. Paulson (ed.), "Regulatory Leeway Sought for Process Analytical Technology. The Gold Sheet", 2002, Vol. 36, Elsevier, Chevy Chase, MD.

2. Process Analytical Technology (PAT) Initiative, U.S. Food and Drug Administration Center for Drug Evaluation and Research Website, http://www.fda.gov/cder/OPS/PAT.htm/.

3. J. P. Higgins, S. M. Arrivo, G. Thurau, R. L. Green, W. Bowen, A. Lange, A. C. Templeton, D. L. Thomas, and R. A. Reed, Anal. Chem., 2003, 75, 1777.

4. H. W. Siesler, Y. Ozaki, S. Kawano, and H. M. Heise, "Near-Infrared Spectroscopy: Principles", 2001, Instruments, Applications, Wiley-VCH, Weinheim.

5. H. Martents and T. Næs, "Multivariate Calibration", 1989, John Wiley \& Sons, New York.

6. B. R. Buchanan, M. A. Baxter, T. S. Chen, X. Z. Qin, and P. A. Robinson, Pharm. Res., 1996, 13, 616.
7. J. K. Drennen and R. A. Lodder, J. Pharm. Sci., 1990, 79, 622.

8. T. Norris, P. K. Aldridge, and S. S. Sekulic, Analyst, 1997, $122,549$.

9. R. W. Saver, P. A. Meulman, D. K. Bowerman, and J. L. Havens, Int. J. Pharm., 1998, 167, 105.

10. A. D. Patl, P. E. Luner, and M. S. Kemper, Int. J. Pharm., 2000, 206, 63.

11. M. Otsuka, F. Kato, and Y. Matsuda, AAPS Pharmsci., 2000, 2, Article 9.

12. M. Otsuka, F. Kato, and Y. Matsuda, Analyst, 2001, 126, 1578.

13. C. R. Bull, Analyst, 1991, 116, 781.

14. L. S. Aucott, P. H. Garthwaite, H. Paul, and S. Buckland, Analyst, 1988, 113, 1849.

15. P. Franke, I. Gill, C. N. Luscombe, D. R. Rudd, J. Waterhouse, and A. Jayasooriya, Analyst, 1998, 123, 2043.

16. K. H. Norris and P. C. Williams, Cereal Chem., 1984, 61, 158.

17. M. Otsuka, Y. Mouri, and Y. Matsuda, AAPS PharmSciTech, 2003, 4(3), Article 42, 334 , http://www.pharmascitech.org.

18. M. Otsuka, Powder Technol., 2004, 141, 244.

19. K. M. Morisseau and C. T. Rhodes, Pharm. Res., 1997, 14, 108.

20. J. D. Kirsch and J. K. Drennen, J. Pharm. Biomed. Anal., 1999, 19, 351.

21. N. K. Ebube, S. S. Thosar, R. A. Roberts, M. S. Kemper, R. Rubnovitz, D. L. Martin, G. E. Reier, T. A. Wheatley, and A. J. Shukala, Pharm. Dev. Technol., 1999, 4, 19.

22. Y. Chen, S. S. Thosar, R. A. Forbess, M. S. Kemper, R. L. Rubinovitz, and A. J. Shukla, Drug Dev. Ind. Pharm., 2001, 27, 623.

23. M. Otsuka and I. Yamane, J. Pharm. Sci., 2006, 95(7), 1425.

24. Pirouette manual, Multivariate Data Analysis for Windows 95/98 and NT, Version 2.6, Infometrix, Inc., Woodinville, WA, USA, http://www.infometrix.com.

25. M. Otsuka, H. Tanabe, K. Osaki, K. Otsuka, and Y. Ozaki, J. Pharm. Sci., 2007 Jan 19. 